Fayetteville State University

DigitalCommons@Fayetteville State University

Math and Computer Science Faculty Working

Papers

Math and Computer Science

2-1-2011

\title{
Additivity Of Jordan (Triple) Derivations On Rings
}

Wu Jing

Fayetteville State University, wjing@uncfsu.edu

Fangyan Lu

Suzhou University

Follow this and additional works at: https://digitalcommons.uncfsu.edu/macsc_wp

\section{Recommended Citation}

Jing, Wu and Lu, Fangyan, "Additivity Of Jordan (Triple) Derivations On Rings" (2011). Math and Computer Science Faculty Working Papers. 5.

https://digitalcommons.uncfsu.edu/macsc_wp/5

This Article is brought to you for free and open access by the Math and Computer Science at DigitalCommons@Fayetteville State University. It has been accepted for inclusion in Math and Computer Science Faculty Working Papers by an authorized administrator of DigitalCommons@Fayetteville State University. For more information, please contact dballar5@uncfsu.edu. 


\title{
ADDITIVITY OF JORDAN (TRIPLE) DERIVATIONS ON RINGS
}

\author{
WU JING AND FANGYAN LU
}

\author{
AbStract. Let $\delta$ be a mapping from ring $R$ into itself satisfying \\ $\delta(a b+b a)=\delta(a) b+a \delta(b)+\delta(b) a+b \delta(a)$ \\ or \\ $\delta(a b a)=\delta(a) b a+a \delta(b)+a b \delta(b)$
}

for all $a, b \in R$. Under some conditions on $R$, we show that $\delta$ is additive.

\section{Introduction and Preliminaries}

In recent years, there has been a great interest in the study of additivity of mappings on rings as well as operator algebras (see [3] - [8], and references therein). Most of these results focus on the additivity of multiplicative maps, Jordan (triple) multiplicative maps, and Jordan elementary maps on rings, triangular algebras, and operator algebras. The first result in this direction is due to Martindale III who obtained the following pioneer result in 1969.

Theorem 1.1. ([8]) Let $R$ be a ring containing a family $\left\{e_{\alpha}: \alpha \in \Lambda\right\}$ of idempotents which satisfies

(1) $x R=\{0\}$ implies $x=0$.

(2) If $e_{\alpha} R x=\{0\}$ for each $\alpha \in \Lambda$, then $x=0$ (and hence $R x=\{0\}$ implies $x=0)$.

(3) For each $\alpha \in \lambda, e_{\alpha} x e_{\alpha} R\left(1-e_{\alpha}\right)=\{0\}$ implies $e_{\alpha} x e_{\alpha}=0$.

Then any multiplicative bijective map from $R$ onto an arbitrary ring $R^{\prime}$ is additive.

Recall that an additive mapping $\delta$ from ring $R$ into itself is called a Jordan derivation if for any $a \in R$

$$
\delta\left(a^{2}\right)=\delta(a) a+a \delta(a),
$$

and $\delta$ is called a Jordan triple derivation if

$$
\delta(a b a)=\delta(a) b a+a \delta(b) a+a b \delta(a)
$$

for any $a, b \in R$. Recently, it has been proved in [6] that if $R$ is a 2-torsion free unital prime ring containing a nontrivial idempotent and $\delta: R \rightarrow R$ is a mapping satisfying

$$
\delta(a b+b a)=\delta(a) b+a \delta(b)+\delta(b) a+b \delta(a)
$$

Date: $11 / 16 / 2010$

2000 Mathematics Subject Classification. 16W25; 16N60; 47B47.

Key words and phrases. Additivity; Jordan derivations; Jordan triple derivations; derivations; idempotents; Peirce decomposition; semiprime rings; prime rings; standard operator algebras.

The first author is supported by Fayetteville State University STEM Program Research Grant. The second author is supported by NNSFC (No. 10771154) and PNSFJ (No. BK2007049). 
or

$$
\delta(a b a)=\delta(a) b a+a \delta(b)+a b \delta(b)
$$

for all $a, b \in R$, then $\delta$ is automatically additive. Moreover, $\delta$ is not only a Jordan (triple) derivation but also a derivation.

The aim of this paper is to generalized the results of [6] to a larger class of rings. Note that our approaches are different from those in [6] which mainly depend on the existence of identity element in the underlying rings.

Let $R$ be an arbitrary ring with a nontrivial idempotent $e$. We write $e_{1}=e$ and $e_{2}=1-e_{1}$. Note that $R$ need not have identity element. Put $e_{i} R e_{j}=R_{i j}$ for any $i, j=1,2$. Then we have the Peirce decomposition of $R$ as $R=R_{11} \oplus R_{12} \oplus R_{21} \oplus$ $R_{22}$. Throughout this paper, the notation $a_{i j}$ will denote an arbitrary element of $R_{i j}$ and any element $a \in R$ can be expressed as $a=a_{11}+a_{12}+a_{21}+a_{22}$.

Let's state our main results.

Theorem 1.2. Let $R$ be a ring containing a nontrivial idempotent and satisfying the following conditions for $i, j, k \in\{1,2\}$ :

(P1) If $a_{i j} x_{j k}=0$ for all $x_{j k} \in R_{j k}$, then $a_{i j}=0$.

(P2) If $x_{i j} a_{j k}=0$ for all $x_{i j} \in R_{i j}$, then $a_{j k}=0$.

(P3) If $a_{i i} x_{i i}+x_{i i} a_{i i}=0$ for all $x_{i i} \in R_{i i}$, then $a_{i i}=0$.

If a mapping $\delta: R \rightarrow R$ satisfies

$$
\delta(a b+b a)=\delta(a) b+a \delta(b)+\delta(b) a+b \delta(a)
$$

for all $a, b \in R$, then $\delta$ is additive.

Theorem 1.3. Suppose $R$ is a ring containing a nontrivial idempotent and satisfies the following conditions:

$\left(P 1^{\prime}\right)$ If $a_{11} x_{12}=0$ for all $x_{12} \in R_{12}$, then $a_{11}=0$.

$\left(P 2^{\prime}\right)$ If $x_{12} a_{22}=0$ for all $x_{12} \in R_{12}$, then $a_{22}=0$.

(P4) If $x_{i j} a x_{i j}=0$ for all $x_{i j} \in R_{i j}(i, j \in\{1,2\})$, then $a_{j i}=0$.

If a mapping $\delta: R \rightarrow R$ satisfies

$$
\delta(a b a)=\delta(a) b a+a \delta(b)+a b \delta(b) \quad \text { for all } a, b \in R,
$$

then $\delta$ is additive.

Obviously, Conditions $\left(\mathrm{P} 1^{\prime}\right)$ and $\left(\mathrm{P} 2^{\prime}\right)$ are special cases of $(\mathrm{P} 1)$ and $(\mathrm{P} 2)$, respectively.

Recall that a ring $R$ is prime if $a R b=\{0\}$ implies that either $a=0$ or $b=0$, and is semiprime if $a R a=\{0\}$ implies $a=0$. We need the following lemma which is due to Brešar.

Lemma 1.4. ([1]) Let $R$ be a 2-torsion free semiprime ring and let $a, b \in R$. If for all $x \in R$ the relation

$$
a x b+b x a=0
$$

holds, then $a x b=b x a=0$ is fulfilled for all $x \in R$.

The following lemmas show that Conditions (P1) to (P4) are fulfilled if $R$ a 2-torsion free semiprime ring under some technical assumptions concerning the nontrivial idempotent or a 2-torsion free prime ring with a nontrivial idempotent.

Lemma 1.5. Let $R$ be a 2-torsion free semiprime ring with a nontrivial idempotent, then 
(P3) If $a_{i i} x_{i i}+x_{i i} a_{i i}=0$ for all $x_{i i} \in R_{i i}$, then $a_{i i}=0$.

In addition, if $R$ satisfies the following conditions:

(C1) If $a_{i i} x_{i j}=0$ for all $x_{i j} \in R_{i j}(i \neq j)$, then $a_{i i}=0$;

(C2) If $x_{j i} a_{i i}=0$ for all $x_{j i} \in R_{j i}(i \neq j)$, then $a_{i i}=0$;

(C3) If $x_{i j} a x_{i j}=0$ for all $x_{i j} \in R_{i j}(i \neq j)$, then $a_{j i}=0$,

then Conditions (P1), (P2), and (P4) are fulfilled, respectively. That is,

(P1) If $a_{i j} x_{j k}=0$ for all $x_{j k} \in R_{j k}$, then $a_{i j}=0$.

(P2) If $x_{i j} a_{j k}=0$ for all $x_{i j} \in R_{i j}$, then $a_{j k}=0$.

(P4) If $x_{i j} a x_{i j}=0$ for all $x_{i j} \in R_{i j}(i, j \in\{1,2\})$, then $a_{j i}=0$.

Proof. (P3) If $i=1$, we rewrite $a_{11} x_{11}+x_{11} a_{11}=0$ as

$$
e_{1} a e_{1} x e_{1}+e_{1} x e_{1} a e_{1}=0 \text { for all } x \in R \text {. }
$$

By Lemma 1.4, we obtain that $e_{1} a e_{1} x e_{1}=0$, which yields that $e_{1} a e_{1} x e_{1} a e_{1}=0$ for all $x \in R$. Since $R$ is semiprime, it follows that $e_{1} a e_{1}=0$. Equivalently, $a_{11}=0$.

Suppose now that $i=2$. We would like to mention here that $e_{2}$ is not necessarily in $R$. Observe that

$$
a_{22} x_{22}+x_{22} a_{22}=0 \quad \text { for all } x_{22} \in R_{22}
$$

is equivalent to

$$
e_{2} a e_{2} x e_{2}+e_{2} x e_{2} a_{2}=0 \quad \text { for all } x \in R \text {. }
$$

Furthermore, for any $x, y \in R$, we have

$$
e_{2} a e_{2} x e_{2} y e_{2}+e_{2} x e_{2} y e_{2} a e_{2}=0,
$$

which implies that

$$
e_{2} a e_{2} x e_{2} y e_{2}=-e_{2} x e_{2} y e_{2} a e_{2} .
$$

Now for any $z \in R$, using the above equality three times we obtain

$$
\begin{aligned}
& \left(e_{2} a e_{2} x e_{2} y e_{2}\right) z e_{2} a e_{2} x e_{2} y e_{2} \\
= & -e_{2} x e_{2} y e_{2} a e_{2} z e_{2} a e_{2} x e_{2} y e_{2} \\
= & -e_{2} x e_{2} y\left(e_{2} a e_{2}\right)\left(e_{2} z e_{2} a e_{2} x e_{2} y e_{2}\right) \\
= & e_{2} x e_{2} y e_{2} z e_{2} a e_{2} x e_{2} y e_{2} a e_{2} \\
= & \left(e_{2} x e_{2} y e_{2} z e_{2} a e_{2} x e_{2} y e_{2}\right)\left(e_{2} a e_{2}\right) \\
= & -e_{2} a e_{2} x e_{2} y e_{2} z e_{2} a e_{2} x e_{2} y e_{2} .
\end{aligned}
$$

Since $R$ is 2 -torsion free, we see that $e_{2} a e_{2} x e_{2} y e_{2}=0$ holds for all $x, y \in R$. And so $e_{2} a e_{2} x e_{2} y e_{2} a e_{2} x e_{2}=0$. By the semiprimeness of $R$ we get $e_{2} a e_{2} x e_{2}=0$ for all $x \in R$. This leads to $e_{2} a e_{2} x e_{2} a e_{2}=0$ for all $x \in R$. Again, using the fact that $R$ is semiprime, we can conclude that $e_{2} a e_{2}=0$, i.e., $a_{22}=0$, as desired.

(P1) In view of Condition (C1), we only need to show the cases of $j=k$ and $j \neq k=i$.

Assume first that $j=k$ and $a_{i j} x_{j j}=0$ for all $x_{j j} \in R_{j j}$. Equivalently, we have $e_{i} a e_{j} x e_{j}$ for all $x \in R$, which implies that $e_{i} a e_{j} x e_{i} a e_{j}$ for all $x \in R$. It follows that $e_{i} a e_{j}=0$ since $R$ is semiprime, that is $a_{i j}=0$.

If $j \neq k=i$ and $a_{i j} x_{j i}=0$ for all $x_{j i} \in R_{j i}$, then $e_{i} a e_{j} x e_{i}=0$ for all $x \in R$. Therefore $e_{i} a e_{j} x e_{i} a e_{j}=0$ for all $x \in R$. Using the fact that $R$ is semiprime, we see that $e_{i} a e_{j}=0$, i.e., $a_{i j}=0$.

(P2) Similar to the proof of (P1). 
(P4) It suffices to show that if $x_{i i} a x_{i i}=0$ for all $x_{i i} \in R_{i i}$ then $a_{i i}=0$. Let $x_{i i}, y_{i i}, z_{i i} \in R_{i i}$ be arbitrary. From $\left(x_{i i}+y_{i i}\right) a\left(x_{i i}+y_{i i}\right)=0$ we can get $x_{i i} a y_{i i}+y_{i i} a x_{i i}=0$ which is equivalent to

$$
x_{i i} a y_{i i}=-y_{i i} a x_{i i}
$$

Applying this equality three times we have

$$
\left(x_{i i} a y_{i i}\right) a z_{i i}=-y_{i i} a\left(x_{i i} a z_{i i}\right)=\left(y_{i i} a z_{i i}\right) a x_{i i}=-x_{i i} a y_{i i} a z_{i i} .
$$

This yields that $x_{i i} a y_{i i} a z_{i i}=0$ since $R$ is 2-torsion free. Particularly,

$$
\left(e_{i} a x_{i i} a e_{i}\right) y\left(e_{i} a x_{i i} a e_{i}\right)=0
$$

for all $y \in R$. It follows that $e_{i} a x_{i i} a e_{i}=0$ for all $x_{i i} \in R_{i i}$ since $R$ is semiprime. Furthermore, we have $\left(e_{i} a e_{i}\right) x\left(e_{i} a e_{i}\right)=0$ for all $x \in R$., and so $e_{i} a e_{i}=0$, i.e., $a_{i i}=0$.

Lemma 1.6. Let $R$ be a 2-torsion free prime ring with a nontrivial idempotent and $i, j, k \in\{1,2\}$. The $R$ satisfies Conditions (P1), (P2), (P3), and (P4), i.e.,

(P1) If $a_{i j} x_{j k}=0$ for all $x_{j k} \in R_{j k}$, then $a_{i j}=0$.

(P2) If $x_{i j} a_{j k}=0$ for all $x_{i j} \in R_{i j}$, then $a_{j k}=0$.

(P3) If $a_{i i} x_{i i}+x_{i i} a_{i i}=0$ for all $x_{i i} \in R_{i i}$, then $a_{i i}=0$.

(P4) If $x_{i j} a x_{i j}=0$ for all $x_{i j} \in R_{i j}$, then $a_{j i}=0$.

Proof. (P1) and (P2) can be deduced easily from the fact that $R$ is a prime ring.

(P3) It follows from Lemma 1.5 directly.

(P4) See Lemma 2 (i) in [3].

We complete this section by recalling the definition of standard operator algebras. Suppose that $X$ is a Banach space. Let $B(X)$ denote the algebra of all bounded linear operators on $X$, and $F(X)$ denote the algebra of all finite rank operators in $B(X)$. A standard operator algebra is any subalgebra of $B(X)$ which contains $F(X)$.

\section{AdDitivity OF JORDAN DERIVATIONS ON RINGS}

Throughout this section, we always assume that $R$ is a ring with a nontrivial idempotent $e_{1}$ and satisfies

(P1) If $a_{i j} x_{j k}=0$ for all $x_{j k} \in R_{j k}$, then $a_{i j}=0$;

(P2) If $x_{i j} a_{j k}=0$ for all $x_{i j} \in R_{i j}$, then $a_{j k}=0$;

(P3) If $a_{i i} x_{i i}+x_{i i} a_{i i}=0$ for all $x_{i i} \in R_{i i}$, then $a_{i i}=0$.

We also assume that mapping $\delta: R \rightarrow R$ satisfies

$$
\delta(a b+b a)=\delta(a) b+a \delta(b)+\delta(b) a+b \delta(a) \quad \text { for all } a, b \in R .
$$

Let's begin with

Lemma 2.1. (1) $\delta\left(a_{11}+b_{12}\right)=\delta\left(a_{11}\right)+\delta\left(b_{12}\right)$.

(2) $\delta\left(a_{11}+b_{21}\right)=\delta\left(a_{11}\right)+\delta\left(b_{21}\right)$.

(3) $\delta\left(a_{22}+b_{12}\right)=\delta\left(a_{22}\right)+\delta\left(b_{12}\right)$.

(4) $\delta\left(a_{22}+b_{21}\right)=\delta\left(a_{22}\right)+\delta\left(b_{21}\right)$. 
Proof. We only prove (1). The rest of the proof goes similarly.

For any $x_{22} \in R_{22}$, we compute

$$
\begin{aligned}
& \delta\left[\left(a_{11}+b_{12}\right) x_{22}+x_{22}\left(a_{11}+b_{12}\right)\right] \\
= & \delta\left(a_{11}+b_{12}\right) x_{22}+\left(a_{11}+b_{12}\right) \delta\left(x_{22}\right)+\delta\left(x_{22}\right)\left(a_{11}+b_{12}\right)+x_{22} \delta\left(a_{11}+b_{12}\right) .
\end{aligned}
$$

On the other hand,

$$
\begin{aligned}
& \delta\left[\left(a_{11}+b_{12}\right) x_{22}+x_{22}\left(a_{11}+b_{12}\right)\right] \\
= & \delta\left(b_{12} x_{22}\right) \\
= & \delta\left(a_{11} x_{22}+x_{22} a_{11}\right)+\delta\left(b_{12} x_{22}+x_{22} b_{12}\right) \\
= & \delta\left(a_{11}\right) x_{22}+a_{11} \delta\left(x_{22}\right)+\delta\left(x_{22}\right) a_{11}+x_{22} \delta\left(a_{11}\right) \\
& +\delta\left(b_{12}\right) x_{22}+b_{12} \delta\left(x_{22}\right)+\delta\left(x_{22}\right) b_{12}+x_{22} \delta\left(b_{12}\right) .
\end{aligned}
$$

Comparing these two equalities we obtain

$$
\left[\delta\left(a_{11}+b_{12}\right)-\delta\left(a_{11}\right)-\delta\left(b_{12}\right)\right] x_{22}+x_{22}\left[\delta\left(a_{11}+b_{12}\right)-\delta\left(a_{11}\right)-\delta\left(b_{12}\right)\right]=0 .
$$

This gives us

$$
\begin{aligned}
& {\left[\delta\left(a_{11}+b_{12}\right)-\delta\left(a_{11}\right)-\delta\left(b_{12}\right)\right]_{12} x_{22}=0} \\
& x_{22}\left[\delta\left(a_{11}+b_{12}\right)-\delta\left(a_{11}\right)-\delta\left(b_{12}\right)\right]_{21}=0
\end{aligned}
$$

and

$$
\left[\delta\left(a_{11}+b_{12}\right)-\delta\left(a_{11}\right)-\delta\left(b_{12}\right)\right]_{22} x_{22}+x_{22}\left[\delta\left(a_{11}+b_{12}\right)-\delta\left(a_{11}\right)-\delta\left(b_{12}\right)\right]_{22}=0 .
$$

By Conditions (P1), (P2), and (P3), we have

$$
\begin{aligned}
& {\left[\delta\left(a_{11}+b_{12}\right)-\delta\left(a_{11}\right)-\delta\left(b_{12}\right)\right]_{12}=0} \\
& {\left[\delta\left(a_{11}+b_{12}\right)-\delta\left(a_{11}\right)-\delta\left(b_{12}\right)\right]_{21}=0} \\
& {\left[\delta\left(a_{11}+b_{12}\right)-\delta\left(a_{11}\right)-\delta\left(b_{12}\right)\right]_{22}=0}
\end{aligned}
$$

In order to complete the proof, we now show that $\left[\delta\left(a_{11}+b_{12}\right)-\delta\left(a_{11}\right)-\right.$ $\left.\delta\left(b_{12}\right)\right]_{11}=0$.

For any $x_{12} \in R_{12}$, note that

$\delta\left[\left(a_{11}+b_{12}\right) x_{12}+x_{12}\left(a_{11}+b_{12}\right)\right]=\delta\left(a_{11} x_{12}\right)=\delta\left(a_{11} x_{12}+x_{12} a_{11}\right)+\delta\left(b_{12} x_{12}+x_{12} b_{12}\right)$.

Applying Equality (1.1) to both sides of this identity, one can deduce that

$$
\left[\delta\left(a_{11}+b_{12}\right)-\delta\left(a_{11}\right)-\delta\left(b_{12}\right)\right] x_{12}+x_{12}\left[\delta\left(a_{11}+b_{12}\right)-\delta\left(a_{11}\right)-\delta\left(b_{12}\right)\right]=0 .
$$

Consequently,

$$
\left[\delta\left(a_{11}+b_{12}\right)-\delta\left(a_{11}\right)-\delta\left(b_{12}\right)\right]_{11} x_{12}=0 .
$$

It follows from Condition $(\mathrm{P} 1)$ that $\left[\delta\left(a_{11}+b_{12}\right)-\delta\left(a_{11}\right)-\delta\left(b_{12}\right)\right]_{11}=0$, which completes the proof.

Lemma 2.2. (1) $\delta\left(a_{12}+b_{12} c_{22}\right)=\delta\left(a_{12}\right)+\delta\left(b_{12} c_{22}\right)$.

(2) $\delta\left(a_{21}+b_{22} c_{21}\right)=\delta\left(a_{21}\right)+\delta\left(b_{22} c_{21}\right)$. 
Proof. (1) Using Lemma 2.1, we obtain

$$
\begin{aligned}
& \delta\left(a_{12}+b_{12} c_{22}\right) \\
= & \delta\left[\left(e_{1}+b_{12}\right)\left(a_{12}+c_{22}\right)+\left(a_{12}+c_{22}\right)\left(e_{1}+b_{12}\right)\right] \\
= & \delta\left(e_{1}+b_{12}\right)\left(a_{12}+c_{22}\right)+\left(e_{1}+b_{12}\right) \delta\left(a_{12}+c_{22}\right) \\
& +\delta\left(a_{12}+c_{22}\right)\left(e_{1}+b_{12}\right)+\left(a_{12}+c_{22}\right) \delta\left(e_{1}+b_{12}\right) \\
= & {\left[\delta\left(e_{1}\right)+\delta\left(b_{12}\right)\right]\left(a_{12}+c_{22}\right)+\left(e_{1}+b_{12}\right)\left[\delta\left(a_{12}\right)+\delta\left(c_{22}\right)\right] } \\
& +\left[\delta\left(a_{12}\right)+\delta\left(c_{22}\right)\right]\left(e_{1}+b_{12}\right)+\left(a_{12}+c_{22}\right)\left[\delta\left(e_{1}\right)+\delta\left(b_{12}\right)\right] \\
= & \delta\left(a_{12}\right)+\delta\left(b_{12} c_{22}\right) .
\end{aligned}
$$

(2) Note that

$$
a_{21}+b_{22} c_{21}=\left(e_{1}+c_{21}\right)\left(a_{21}+b_{22}\right)+\left(a_{21}+b_{22}\right)\left(e_{1}+c_{21}\right) .
$$

Now the proof goes similarly to that of (1).

Lemma 2.3. (1) $\delta\left(a_{12}+b_{12}\right)=\delta\left(a_{12}\right)+\delta\left(b_{12}\right)$.

(2) $\delta\left(a_{21}+b_{21}\right)=\delta\left(a_{21}\right)+\delta\left(b_{21}\right)$.

Proof. We only prove (1). The proof of (2) is similar.

For any $x_{22} \in R_{22}$, we calculate $\delta\left[\left(a_{12}+b_{12}\right) x_{22}+x_{22}\left(a_{12}+b_{12}\right)\right]$ in two ways.

On one hand,

$$
\begin{aligned}
& \delta\left[\left(a_{12}+b_{12}\right) x_{22}+x_{22}\left(a_{12}+b_{12}\right)\right] \\
= & \delta\left(a_{12}+b_{12}\right) x_{22}+\left(a_{12}+b_{12}\right) \delta\left(x_{22}\right)+\delta\left(x_{22}\right)\left(a_{12}+b_{12}\right)+x_{22} \delta\left(a_{12}+b_{12}\right) .
\end{aligned}
$$

On the other hand, by Lemma 2.2

$$
\begin{aligned}
& \delta\left[\left(a_{12}+b_{12}\right) x_{22}+x_{22}\left(a_{12}+b_{12}\right)\right] \\
= & \delta\left(a_{12} x_{22}+b_{12} x_{22}\right) \\
= & \delta\left(a_{12} x_{22}\right)+\delta\left(b_{12} x_{22}\right) \\
= & \delta\left(a_{12} x_{22}+x_{22} a_{12}\right)+\delta\left(b_{12} x_{22}+x_{22} b_{12}\right) \\
= & \delta\left(a_{12}\right) x_{22}+a_{12} \delta\left(x_{22}\right)+\delta\left(x_{22}\right) a_{12}+x_{22} \delta\left(a_{12}\right) \\
& \delta\left(b_{12}\right) x_{22}+b_{12} \delta\left(x_{22}\right)+\delta\left(x_{22}\right) b_{12}+x_{22} \delta\left(b_{12}\right) .
\end{aligned}
$$

These give us

$$
\left[\delta\left(a_{12}+b_{12}\right)-\delta\left(a_{12}\right)-\delta\left(b_{12}\right)\right] x_{22}+x_{22}\left[\delta\left(a_{12}+b_{12}\right)-\delta\left(a_{12}\right)-\delta\left(b_{12}\right)\right]=0
$$

for all $x_{22} \in R_{22}$.

It follows that

$$
\begin{aligned}
& {\left[\delta\left(a_{12}+b_{12}\right)-\delta\left(a_{12}\right)-\delta\left(b_{12}\right)\right]_{12} x_{22}=0} \\
& x_{22}\left[\delta\left(a_{12}+b_{12}\right)-\delta\left(a_{12}\right)-\delta\left(b_{12}\right)\right]_{21}=0
\end{aligned}
$$

and

$$
\left[\delta\left(a_{12}+b_{12}\right)-\delta\left(a_{12}\right)-\delta\left(b_{12}\right)\right]_{22} x_{22}+x_{22}\left[\delta\left(a_{12}+b_{12}\right)-\delta\left(a_{12}\right)-\delta\left(b_{12}\right)\right]_{22}=0 .
$$

By Conditions (P1), (P2), and (P3), we can deduce that

$$
\begin{aligned}
& {\left[\delta\left(a_{12}+b_{12}\right)-\delta\left(a_{12}\right)-\delta\left(b_{12}\right)\right]_{12}=0} \\
& {\left[\delta\left(a_{12}+b_{12}\right)-\delta\left(a_{12}\right)-\delta\left(b_{12}\right)\right]_{21}=0} \\
& {\left[\delta\left(a_{12}+b_{12}\right)-\delta\left(a_{12}\right)-\delta\left(b_{12}\right)\right]_{22}=0 .}
\end{aligned}
$$


We now complete the proof by showing that

$$
\left[\delta\left(a_{12}+b_{12}\right)-\delta\left(a_{12}\right)-\delta\left(b_{12}\right)\right]_{11}=0 .
$$

To this end, for any $x_{12} \in R_{12}$, we compute

$$
\begin{aligned}
& \delta\left(a_{12}+b_{12}\right) x_{12}+\left(a_{12}+b_{12}\right) \delta\left(x_{12}\right)+\delta\left(x_{12}\right)\left(a_{12}+b_{12}\right)+x_{12} \delta\left(a_{12}+b_{12}\right) \\
= & \delta\left[\left(a_{12}+b_{12}\right) x_{12}+x_{12}\left(a_{12}+b_{12}\right)\right] \\
= & 0 \\
= & \delta\left(a_{12} x_{12}+x_{12} a_{12}\right)+\delta\left(b_{12} x_{12}+x_{12} b_{12}\right) \\
= & \delta\left(a_{12}\right) x_{12}+a_{12} \delta\left(x_{12}\right)+\delta\left(x_{12}\right) a_{12}+x_{12} \delta\left(a_{12}\right) \\
& +\delta\left(b_{12}\right) x_{12}+b_{12} \delta\left(x_{12}\right)+\delta\left(x_{12}\right) b_{12}+x_{12} \delta\left(b_{12}\right) .
\end{aligned}
$$

This yields that

$$
\left[\delta\left(a_{12}+b_{12}\right)-\delta\left(a_{12}\right)-\delta\left(b_{12}\right)\right] x_{12}+x_{12}\left[\delta\left(a_{12}+b_{12}\right)-\delta\left(a_{12}\right)-\delta\left(b_{12}\right)\right]=0 .
$$

Therefore, we have

$$
\left[\delta\left(a_{12}+b_{12}\right)-\delta\left(a_{12}\right)-\delta\left(b_{12}\right)\right]_{11} x_{12}=0 \quad \text { for all } x_{12} \in R_{12} .
$$

By Condition (P1) we can infer that $\left[\delta\left(a_{12}+b_{12}\right)-\delta\left(a_{12}\right)-\delta\left(b_{12}\right)\right]_{11}=0$, which completes the proof.

Lemma 2.4. (1) $\delta\left(a_{11}+b_{11}\right)=\delta\left(a_{11}\right)+\delta\left(b_{11}\right)$.

(2) $\delta\left(a_{22}+b_{22}\right)=\delta\left(a_{22}\right)+\delta\left(b_{22}\right)$.

Proof. We only prove (1). For any $x_{22} \in R_{22}$, we have

$$
\begin{aligned}
& \delta\left(a_{11}+b_{11}\right) x_{22}+\left(a_{11}+b_{11}\right) \delta\left(x_{22}\right)+\delta\left(x_{22}\right)\left(a_{11}+b_{11}\right)+x_{22} \delta\left(a_{11}+b_{11}\right) \\
= & \delta\left[\left(a_{11}+b_{11}\right) x_{22}+x_{22}\left(a_{11}+b_{11}\right)\right] \\
= & 0 \\
= & \delta\left(a_{11} x_{22}+x_{22} a_{11}\right)+\delta\left(b_{11} x_{22}+x_{22} b_{11}\right) \\
= & \delta\left(a_{11}\right) x_{22}+a_{11} \delta\left(x_{22}\right)+\delta\left(x_{22}\right) a_{11}+x_{22} \delta\left(a_{11}\right) \\
& +\delta\left(b_{11}\right) x_{22}+b_{11} \delta\left(x_{22}\right)+\delta\left(x_{22}\right) b_{11}+x_{22} \delta\left(b_{11}\right) .
\end{aligned}
$$

This gives us

$$
\left[\delta\left(a_{11}+b_{11}\right)-\delta\left(a_{11}\right)-\delta\left(b_{11}\right)\right] x_{22}+x_{22}\left[\delta\left(a_{11}+b_{11}\right)-\delta\left(a_{11}\right)-\delta\left(b_{11}\right)\right]=0,
$$

which implies that

$$
\begin{aligned}
& {\left[\delta\left(a_{11}+b_{11}\right)-\delta\left(a_{11}\right)-\delta\left(b_{11}\right)\right]_{12}=0,} \\
& {\left[\delta\left(a_{11}+b_{11}\right)-\delta\left(a_{11}\right)-\delta\left(b_{11}\right)\right]_{21}=0,} \\
& {\left[\delta\left(a_{11}+b_{11}\right)-\delta\left(a_{11}\right)-\delta\left(b_{11}\right)\right]_{22}=0 .}
\end{aligned}
$$

Similarly, by considering $\left(a_{11}+b_{11}\right) x_{12}+x_{12}\left(a_{11}+b_{11}\right)$ and using Lemma 2.3 one can deduce that $\left[\delta\left(a_{11}+b_{11}\right)-\delta\left(a_{11}\right)-\delta\left(b_{11}\right)\right]_{11}=0$.

Lemma 2.5. $\delta\left(a_{12}+b_{21}\right)=\delta\left(a_{12}\right)+\delta\left(b_{21}\right)$.

Proof. From $a_{12}+b_{21}=\left(a_{12}+b_{21}\right) e_{1}+e_{1}\left(a_{12}+b_{21}\right)$ we have

$$
\begin{aligned}
& \delta\left(a_{12}+b_{21}\right) \\
= & \delta\left(a_{12}+b_{21}\right) e_{1}+\left(a_{12}+b_{21}\right) \delta\left(e_{1}\right)+\delta\left(e_{1}\right)\left(a_{12}+b_{21}\right)+e_{1} \delta\left(a_{12}+b_{21}\right) .
\end{aligned}
$$


Multiplying this equality from right by $e_{1}$ we arrive at

$$
0=\left(a_{12}+b_{21}\right) \delta\left(e_{1}\right) e_{1}+\delta\left(e_{1}\right) b_{21}+e_{1} \delta\left(a_{12}+b_{21}\right) e_{1} .
$$

Similarly we can obtain

$$
\begin{aligned}
& 0=a_{12} \delta\left(e_{1}\right) e_{1}+e_{1} \delta\left(a_{12}\right) e_{1} \\
& 0=b_{21} \delta\left(e_{1}\right) e_{1}+\delta\left(e_{1}\right) b_{21}+e_{1} \delta\left(b_{21}\right) e_{1} .
\end{aligned}
$$

Comparing the above three equalities we see that

$$
\left[\delta\left(a_{12}+b_{21}\right)-\delta\left(a_{12}\right)-\delta\left(b_{21}\right)\right]_{11}=e_{1}\left[\delta\left(a_{12}+b_{21}\right)-\delta\left(a_{12}\right)-\delta\left(b_{21}\right)\right] e_{1}=0 .
$$

Now, for any $x_{12} \in R_{12}$, we have

$$
\begin{aligned}
& \delta\left(a_{12}+b_{21}\right) x_{12}+\left(a_{12}+b_{21}\right) \delta\left(x_{12}\right)+\delta\left(x_{12}\right)\left(a_{12}+b_{21}\right)+x_{12} \delta\left(a_{12}+b_{21}\right) \\
= & \delta\left[\left(a_{12}+b_{21}\right) x_{12}+x_{12}\left(a_{12}+b_{21}\right)\right] \\
= & \delta\left(b_{21} x_{12}+x_{12} b_{21}\right) \\
= & \delta\left(b_{21} x_{12}+x_{12} b_{21}\right)+\delta\left(a_{12} x_{12}+x_{12} a_{12}\right) \\
= & \delta\left(b_{21}\right) x_{12}+b_{21} \delta\left(x_{12}\right)+\delta\left(x_{12}\right) b_{21}+x_{12} \delta\left(b_{21}\right) \\
& +\delta\left(a_{12}\right) x_{12}+a_{12} \delta\left(x_{12}\right)+\delta\left(x_{12}\right) a_{12}+x_{12} \delta\left(a_{12}\right),
\end{aligned}
$$

which leads to

$$
\left[\delta\left(a_{12}+b_{21}\right)-\delta\left(a_{12}\right)-\delta\left(b_{21}\right)\right] x_{12}+x_{12}\left[\delta\left(a_{12}+b_{21}\right)-\delta\left(a_{12}\right)-\delta\left(b_{21}\right)\right]=0 .
$$

Since $\left[\delta\left(a_{12}+b_{21}\right)-\delta\left(a_{12}\right)-\delta\left(b_{21}\right)\right]_{11}=0$, we see that

$$
\begin{aligned}
& {\left[\delta\left(a_{12}+b_{21}\right)-\delta\left(a_{12}\right)-\delta\left(b_{21}\right)\right]_{21} x_{12}=0,} \\
& x_{12}\left[\delta\left(a_{12}+b_{21}\right)-\delta\left(a_{12}\right)-\delta\left(b_{21}\right)\right]_{22}=0 .
\end{aligned}
$$

It follows from Conditions (P1) and (P2) that

$$
\left[\delta\left(a_{12}+b_{21}\right)-\delta\left(a_{12}\right)-\delta\left(b_{21}\right)\right]_{21}=\left[\delta\left(a_{12}+b_{21}\right)-\delta\left(a_{12}\right)-\delta\left(b_{21}\right)\right]_{22}=0 .
$$

Similarly, by considering $\delta\left[\left(a_{12}+b_{21}\right) x_{21}+x_{21}\left(a_{12}+b_{21}\right)\right]$ for all $x_{21} \in R_{21}$, we can get

$$
\left[\delta\left(a_{12}+b_{21}\right)-\delta\left(a_{12}\right)-\delta\left(b_{21}\right)\right] x_{21}+x_{21}\left[\delta\left(a_{12}+b_{21}\right)-\delta\left(a_{12}\right)-\delta\left(b_{21}\right)\right]=0 .
$$

Consequently, $\left[\delta\left(a_{12}+b_{21}\right)-\delta\left(a_{12}\right)-\delta\left(b_{21}\right)\right]_{12}=0$.

Lemma 2.6. (1) $\delta\left(a_{11}+b_{12}+c_{21}\right)=\delta\left(a_{11}\right)+\delta\left(b_{12}\right)+\delta\left(c_{21}\right)$.

(2) $\delta\left(a_{12}+b_{21}+c_{22}\right)=\delta\left(a_{12}\right)+\delta\left(b_{21}\right)+\delta\left(c_{22}\right)$.

Proof. We only prove (1). For any $x_{22} \in R_{22}$, we have

$$
\begin{aligned}
& \delta\left[\left(a_{11}+b_{12}+c_{21}\right) x_{22}+x_{22}\left(a_{11}+b_{12}+c_{21}\right)\right] \\
=\quad & \delta\left(a_{11}+b_{12}+c_{21}\right) x_{22}+\left(a_{11}+b_{12}+c_{21}\right) \delta\left(x_{22}\right) \\
+ & \delta\left(x_{22}\right)\left(a_{11}+b_{12}+c_{21}\right)+x_{22} \delta\left(a_{11}+b_{12}+c_{21}\right) .
\end{aligned}
$$


On the other hand, by Lemma 2.5, we also have

$$
\begin{aligned}
& \delta\left[\left(a_{11}+b_{12}+c_{21}\right) x_{22}+x_{22}\left(a_{11}+b_{12}+c_{21}\right)\right] \\
= & \delta\left(b_{12} x_{22}+x_{22} c_{21}\right) \\
= & \delta\left(b_{12} x_{22}\right)+\delta\left(x_{22} c_{21}\right) \\
= & \delta\left(a_{11} x_{22}+x_{22} a_{11}\right)+\delta\left(b_{12} x_{22}+x_{22} b_{12}\right)+\delta\left(c_{21} x_{22}+x_{22} c_{21}\right) \\
= & \delta\left(a_{11}\right) x_{22}+a_{11} \delta\left(x_{22}\right)+\delta\left(x_{22}\right) a_{11}+x_{22} \delta\left(a_{11}\right) \\
& +\delta\left(b_{12}\right) x_{22}+b_{12} \delta\left(x_{22}\right)+\delta\left(x_{22}\right) b_{12}+x_{22} \delta\left(b_{12}\right) \\
& +\delta\left(c_{21}\right) x_{22}+c_{21} \delta\left(x_{22}\right)+\delta\left(x_{22}\right) c_{21}+x_{22} \delta\left(c_{21}\right) .
\end{aligned}
$$

It follows that

$$
\begin{aligned}
& {\left[\delta\left(a_{11}+b_{12}+c_{21}\right)-\delta\left(a_{11}\right)-\delta\left(b_{12}\right)-\delta\left(c_{21}\right)\right] x_{22}} \\
& +x_{22}\left[\delta\left(a_{11}+b_{12}+c_{21}\right)-\delta\left(a_{11}\right)-\delta\left(b_{12}\right)-\delta\left(c_{21}\right)\right]=0 .
\end{aligned}
$$

Then we can obtain that

$$
\begin{aligned}
& {\left[\delta\left(a_{11}+b_{12}+c_{21}\right)-\delta\left(a_{11}\right)-\delta\left(b_{12}\right)-\delta\left(c_{21}\right)\right]_{12}=0} \\
& {\left[\delta\left(a_{11}+b_{12}+c_{21}\right)-\delta\left(a_{11}\right)-\delta\left(b_{12}\right)-\delta\left(c_{21}\right)\right]_{21}=0} \\
& {\left[\delta\left(a_{11}+b_{12}+c_{21}\right)-\delta\left(a_{11}\right)-\delta\left(b_{12}\right)-\delta\left(c_{21}\right)\right]_{22}=0}
\end{aligned}
$$

From

$$
\begin{aligned}
& \delta\left[\left(a_{11}+b_{12}+c_{21}\right) x_{12}+x_{12}\left(a_{11}+b_{12}+c_{21}\right)\right] \\
= & \delta\left[\left(a_{11}+c_{21}\right) x_{12}+x_{12}\left(a_{11}+c_{21}\right)\right]+\delta\left(b_{12} x_{12}+x_{12} b_{12}\right)
\end{aligned}
$$

and using Lemma 2.1, one can easily get

$$
\begin{aligned}
& {\left[\delta\left(a_{11}+b_{12}+c_{21}\right)-\delta\left(a_{11}\right)-\delta\left(b_{12}\right)-\delta\left(c_{21}\right)\right] x_{12}} \\
& +x_{12}\left[\delta\left(a_{11}+b_{12}+c_{21}\right)-\delta\left(a_{11}\right)-\delta\left(b_{12}\right)-\delta\left(c_{21}\right)\right]=0 .
\end{aligned}
$$

It follows that $\left[\delta\left(a_{11}+b_{12}+c_{21}\right)-\delta\left(a_{11}\right)-\delta\left(b_{12}\right)-\delta\left(c_{21}\right)\right]_{11} x_{12}=0$, and so

$$
\left[\delta\left(a_{11}+b_{12}+c_{21}\right)-\delta\left(a_{11}\right)-\delta\left(b_{12}\right)-\delta\left(c_{21}\right)\right]_{11}=0 .
$$

Lemma 2.7. $\delta\left(a_{11}+b_{12}+c_{21}+d_{22}\right)=\delta\left(a_{11}\right)+\delta\left(b_{12}\right)+\delta\left(c_{21}\right)+\delta\left(d_{22}\right)$.

Proof. For any $x_{11} \in R_{11}$, by Lemma 2.6, we have

$$
\begin{aligned}
& \delta\left(a_{11}+b_{12}+c_{21}+d_{22}\right) x_{11}+\left(a_{11}+b_{12}+c_{21}+d_{22}\right) \delta\left(x_{11}\right) \\
& +\delta\left(x_{11}\right)\left(a_{11}+b_{12}+c_{21}+d_{22}\right)+x_{11} \delta\left(a_{11}+b_{12}+c_{21}+d_{22}\right] \\
= & \delta\left[\left(a_{11}+b_{12}+c_{21}+d_{22}\right) x_{11}+x_{11}\left(a_{11}+b_{12}+c_{21}+d_{22}\right)\right] \\
= & \delta\left(a_{11} x_{11}+c_{21} x_{11}+x_{11} a_{11}+x_{11} b_{12}\right) \\
= & \delta\left(a_{11} x_{11}+x_{11} a_{11}\right)+\delta\left(x_{11} b_{12}\right)+\delta\left(c_{21} x_{11}\right) \\
= & \delta\left(a_{11} x_{11}+x_{11} a_{11}\right)+\delta\left(b_{12} x_{11}+b_{12} x_{11}\right) \\
& +\delta\left(c_{21} x_{11}+x_{11} c_{21}\right)+\delta\left(d_{22} x_{11}+x_{11} d_{22}\right) .
\end{aligned}
$$

This gives us

$$
\begin{aligned}
& {\left[\delta\left(a_{11}+b_{12}+c_{21}+d_{22}\right)-\delta\left(a_{11}\right)-\delta\left(b_{12}\right)-\delta\left(c_{21}\right)-\delta\left(d_{22}\right)\right] x_{11}} \\
& +x_{11}\left[\delta\left(a_{11}+b_{12}+c_{21}+d_{22}\right)-\delta\left(a_{11}\right)-\delta\left(b_{12}\right)-\delta\left(c_{21}\right)-\delta\left(d_{22}\right)\right]=0 .
\end{aligned}
$$


We can infer that

$$
\begin{aligned}
& {\left[\delta\left(a_{11}+b_{12}+c_{21}+d_{22}\right)-\delta\left(a_{11}\right)-\delta\left(b_{12}\right)-\delta\left(c_{21}\right)-\delta\left(d_{22}\right)\right]_{11}=0} \\
& {\left[\delta\left(a_{11}+b_{12}+c_{21}+d_{22}\right)-\delta\left(a_{11}\right)-\delta\left(b_{12}\right)-\delta\left(c_{21}\right)-\delta\left(d_{22}\right)\right]_{12}=0} \\
& {\left[\delta\left(a_{11}+b_{12}+c_{21}+d_{22}\right)-\delta\left(a_{11}\right)-\delta\left(b_{12}\right)-\delta\left(c_{21}\right)-\delta\left(d_{22}\right)\right]_{21}=0}
\end{aligned}
$$

Similarly, one can get

$$
\left[\delta\left(a_{11}+b_{12}+c_{21}+d_{22}\right)-\delta\left(a_{11}\right)-\delta\left(b_{12}\right)-\delta\left(c_{21}\right)-\delta\left(d_{22}\right)\right]_{22}=0,
$$

which completes the proof.

Now we are ready to prove our first result.

Theorem 2.8. Let $R$ be a ring with a nontrivial idempotent and satisfy

(P1) If $a_{i j} x_{j k}=0$ for all $x_{j k} \in R_{j k}$, then $a_{i j}=0$;

(P2) If $x_{i j} a_{j k}=0$ for all $x_{i j} \in R_{i j}$, then $a_{j k}=0$;

(P3) If $a_{i i} x_{i i}+x_{i i} a_{i i}=0$ for all $x_{i i} \in R_{i i}$, then $a_{i i}=0$,

for $i, j, k \in\{1,2\}$. If a mapping $\delta: R \rightarrow R$ satisfies

$$
\delta(a b+b a)=\delta(a) b+a \delta(b)+\delta(b) a+b \delta(a)
$$

for all $a, b \in R$, then $\delta$ is additive.

In addition, if $R$ is 2-torsion free, then $\delta$ is a Jordan derivation.

Proof. For any $a, b \in R$, we write $a=a_{11}+a_{12}+a_{21}+a_{22}$ and $b=b_{11}+b_{12}+b_{21}+b_{22}$. Applying Lemmas $2.3-2.7$, we have

$$
\begin{aligned}
& \delta(a+b) \\
= & \delta\left(a_{11}+a_{12}+a_{21}+a_{22}+b_{11}+b_{12}+b_{21}+b_{22}\right) \\
= & \delta\left[\left(a_{11}+b_{11}\right)+\left(a_{12}+b_{12}\right)+\left(a_{21}+b_{21}\right)+\left(a_{22}+b_{22}\right)\right] \\
= & \delta\left(a_{11}+b_{11}\right)+\delta\left(a_{12}+b_{12}\right)+\delta\left(a_{21}+b_{21}\right)+\delta\left(a_{22}+b_{22}\right) \\
= & \delta\left(a_{11}\right)+\delta\left(b_{11}\right)+\delta\left(a_{12}\right)+\delta\left(b_{12}\right)+\delta\left(a_{21}\right)+\delta\left(b_{21}\right)+\delta\left(a_{22}\right)+\delta\left(b_{22}\right) \\
= & \delta\left(a_{11}+a_{12}+a_{21}+a_{22}\right)+\delta\left(b_{11}+b_{12}+b_{21}+b_{22}\right) \\
= & \delta(a)+\delta(b),
\end{aligned}
$$

i. e., $\delta$ is additive.

In addition, if $R$ is 2-torsion free, then for any $a \in R$, we have

$$
2 \delta\left(a^{2}\right)=\delta\left(2 a^{2}\right)=\delta(a a+a a)=2[\delta(a) a+a \delta(a)] .
$$

Therefore, $\delta$ is a Jordan derivation.

Applying Lemma 1.5 and the well-known result that every Jordan derivation on a 2-torsion free semiprime ring is a derivation (see [1]), we have

Corollary 2.9. Let $R$ be a 2-torsion free semiprime ring with a nontrivial idempotent satisfying

(C1) If $a_{i i} x_{i j}=0$ for all $x_{i j} \in R_{i j}(i \neq j)$, then $a_{i i}=0$;

(C2) If $x_{j i} a_{i i}=0$ for all $x_{j i} \in R_{j i}(i \neq j)$, then $a_{i i}=0$.

If mapping $\delta: R \rightarrow R$ satisfies

$$
\delta(a b+b a)=\delta(a) b+a \delta(b)+\delta(b) a+b \delta(a)
$$

for all $a, b \in R$, then $\delta$ is additive. Moreover, $\delta$ is a derivation.

As a consequence of Lemma 1.6 we can easily have the following result. 
Corollary 2.10. Let $R$ be a 2-torsion free prime ring containing a nontrivial idempotent and mapping $\delta: R \rightarrow R$ satisfy

$$
\delta(a b+b a)=\delta(a) b+a \delta(b)+\delta(b) a+b \delta(a)
$$

for all $a, b \in R$, then $\delta$ is additive. Moreover, $\delta$ is a derivation.

Since every standard operator algebra is prime, we can easily have

Corollary 2.11. Let $\mathcal{A}$ be a standard operator algebra in a Banach space $X$ whose dimension is greater than 1 . Suppose that $\delta: \mathcal{A} \rightarrow \mathcal{A}$ is a mapping satisfying

$$
\delta(A B+B A)=\delta(A) B+A \delta(B)+\delta(B) A+B \delta(A) \quad \text { for all } A, B \in \mathcal{A},
$$

then $\delta$ is additive. Moreover, $\delta$ is an additive derivation.

\section{Additivity of Jordan triple Derivations On Rings}

The aim of this section is to show that if a mapping $\delta$ from a ring $R$ containing a nontrivial idempotent into itself satisfies Equality (1.2) then $\delta$ is automatically additive.

In what follows we shall assume that $R$ is a ring with a nontrivial idempotent $e_{1}$ satisfying

$\left(\mathrm{P}^{\prime}\right)$ If $a_{11} x_{12}=0$ for all $x_{12} \in R_{12}$, then $a_{11}=0$.

$\left(\mathrm{P}^{\prime}\right)$ If $x_{12} a_{22}=0$ for all $x_{12} \in R_{12}$, then $a_{22}=0$.

(P4) If $x_{i j} a x_{i j}=0$ for all $x_{i j} \in R_{i j}(i, j \in\{1,2\})$, then $a_{j i}=0$.

and $\delta: R \rightarrow R$ is a mapping with the property that

$$
\delta(a b a)=\delta(a) b a+a \delta(b) a+a b \delta(a)
$$

holds true for all $a, b \in R$.

Lemma 3.1. $\delta\left(a_{11}+b_{12}+c_{21}+d_{22}\right)=\delta\left(a_{11}\right)+\delta\left(b_{12}\right)+\delta\left(c_{21}\right)+\delta\left(d_{22}\right)$.

Proof. For any $x_{i j} \in R, i, j=1,2$, on one hand, we have

$$
\begin{aligned}
& \delta\left[x_{i j}\left(a_{11}+b_{12}+c_{21}+d_{22}\right) x_{i j}\right] \\
= & \delta\left(x_{i j}\right)\left(a_{11}+b_{12}+c_{21}+d_{22}\right) x_{i j} \\
& +x_{i j} \delta\left(a_{11}+b_{12}+c_{21}+d_{22}\right) x_{i j} \\
& +x_{i j}\left(a_{11}+b_{12}+c_{21}+d_{22}\right) \delta\left(x_{i j}\right) .
\end{aligned}
$$

On the other hand,

$$
\begin{aligned}
& \delta\left(x_{i j} a_{11} x_{i j}\right)=\delta\left(x_{i j}\right) a_{11} x_{i j}+x_{i j} \delta\left(a_{11}\right) x_{i j}+x_{i j} a_{11} \delta\left(x_{i j}\right), \\
& \delta\left(x_{i j} b_{12} x_{i j}\right)=\delta\left(x_{i j}\right) b_{12} x_{i j}+x_{i j} \delta\left(b_{12}\right) x_{i j}+x_{i j} b_{12} \delta\left(x_{i j}\right), \\
& \delta\left(x_{i j} c_{21} x_{i j}\right)=\delta\left(x_{i j}\right) c_{21} x_{i j}+x_{i j} \delta\left(c_{21}\right) x_{i j}+x_{i j} c_{21} \delta\left(x_{i j}\right), \\
& \delta\left(x_{i j} d_{22} x_{i j}\right)=\delta\left(x_{i j}\right) d_{22} x_{i j}+x_{i j} \delta\left(d_{22}\right) x_{i j}+x_{i j} d_{22} \delta\left(x_{i j}\right) .
\end{aligned}
$$

These imply that

$$
\begin{aligned}
& \delta\left[x_{i j}\left(a_{11}+b_{12}+c_{21}+d_{22}\right) x_{i j}\right]-\delta\left(x_{i j} a_{11} x_{i j}\right) \\
& -\delta\left(x_{i j} b_{12} x_{i j}\right)-\delta\left(x_{i j} c_{21} x_{i j}\right)-\delta\left(x_{i j} d_{22} x_{i j}\right) \\
= & x_{i j}\left[\delta\left(a_{11}+b_{12}+c_{21}+d_{22}\right)-\delta\left(a_{11}\right)-\delta\left(b_{12}\right)-\delta\left(c_{21}\right)-\delta\left(d_{22}\right)\right] x_{i j} .
\end{aligned}
$$


Note that for any $i, j=1,2$, we have

$$
\begin{aligned}
& \delta\left[x_{i j}\left(a_{11}+b_{12}+c_{21}+d_{22}\right) x_{i j}\right]-\delta\left(x_{i j} a_{11} x_{i j}\right) \\
& -\delta\left(x_{i j} b_{12} x_{i j}\right)-\delta\left(x_{i j} c_{21} x_{i j}\right)-\delta\left(x_{i j} d_{22} x_{i j}\right)=0 .
\end{aligned}
$$

Then, for $i, j=1,2$, we get

$$
x_{i j}\left[\delta\left(a_{11}+b_{12}+c_{21}+d_{22}\right)-\delta\left(a_{11}\right)-\delta\left(b_{12}\right)-\delta\left(c_{21}\right)-\delta\left(d_{22}\right)\right] x_{i j}=0
$$

By Condition (P4), we see that

$$
\left[\delta\left(a_{11}+b_{12}+c_{21}+d_{22}\right)-\delta\left(a_{11}\right)-\delta\left(b_{12}\right)-\delta\left(c_{21}\right)-\delta\left(d_{22}\right)\right]_{j i}=0, \quad i, j=1,2 .
$$

Equivalently,

$$
\delta\left(a_{11}+b_{12}+c_{21}+d_{22}\right)-\delta\left(a_{11}\right)-\delta\left(b_{12}\right)-\delta\left(c_{21}\right)-\delta\left(d_{22}\right)=0 .
$$

Lemma 3.2. (1) $\delta\left(a_{12}+b_{12} c_{22}\right)=\delta\left(a_{12}\right)+\delta\left(b_{12} c_{22}\right)$.

(2) $\delta\left(a_{21}+b_{22} c_{21}\right)=\delta\left(a_{21}\right)+\delta\left(b_{22} c_{21}\right)$.

Proof. (1) We first note that

$$
e_{1}+a_{12}+b_{12} c_{22}=\left(e_{1}+a_{12}+c_{22}\right)\left(e_{1}+b_{12}\right)\left(e_{1}+a_{12}+c_{22}\right) \text {. }
$$

By applying Lemma 3.1, we have

$$
\begin{aligned}
& \delta\left(e_{1}\right)+\delta\left(a_{12}+b_{12} c_{22}\right) \\
= & \delta\left(e_{1}+a_{12}+b_{12} c_{22}\right) \\
= & \delta\left[\left(e_{1}+a_{12}+c_{22}\right)\left(e_{1}+b_{12}\right)\left(e_{1}+a_{12}+c_{22}\right)\right] \\
= & \delta\left(e_{1}+a_{12}+c_{22}\right)\left(e_{1}+b_{12}\right)\left(e_{1}+a_{12}+c_{22}\right) \\
& +\left(e_{1}+a_{12}+c_{22}\right) \delta\left(e_{1}+b_{12}\right)\left(e_{1}+a_{12}+c_{22}\right) \\
& +\left(e_{1}+a_{12}+c_{22}\right)\left(e_{1}+b_{12}\right) \delta\left(e_{1}+a_{12}+c_{22}\right) \\
= & \left.\delta\left(e_{1}\right)+\delta\left(a_{12}\right)+\delta\left(c_{22}\right)\right]\left(e_{1}+b_{12}\right)\left(e_{1}+a_{12}+c_{22}\right) \\
& +\left(e_{1}+a_{12}+c_{22}\right)\left[\delta\left(e_{1}\right)+\delta\left(b_{12}\right)\right]\left(e_{1}+a_{12}+c_{22}\right) \\
& +\left(e_{1}+a_{12}+c_{22}\right)\left(e_{1}+b_{12}\right)\left[\delta\left(e_{1}\right)+\delta\left(a_{12}\right)+\delta\left(c_{22}\right)\right] \\
= & \left.\delta\left(e_{1}\right)+\delta\left(a_{12}\right)+\delta\left(c_{22}\right)\right] e_{1}\left(e_{1}+a_{12}+c_{22}\right)+\left(e_{1}+a_{12}+c_{22}\right) \delta\left(e_{1}\right)\left(e_{1}+a_{12}+c_{22}\right) \\
& +\left(e_{1}+a_{12}+c_{22}\right) e_{1}\left[\delta\left(e_{1}\right)+\delta\left(a_{12}\right)+\delta\left(c_{22}\right)\right]+\left[\delta\left(e_{1}\right)+\delta\left(c_{22}\right)\right] b_{12}\left(e_{1}+c_{22}\right) \\
+ & \left(e_{1}+c_{22}\right) \delta\left(b_{12}\right)\left(e_{1}+c_{22}\right)+\left(e_{1}+c_{22}\right) b_{12}\left[\delta\left(e_{1}\right)+\delta\left(c_{22}\right)\right] \\
+ & {\left[\delta\left(a_{12}\right)+\delta\left(c_{22}\right)\right]\left(e_{1}+b_{12}\right)\left(a_{12}+c_{22}\right)+\left(a_{12}+c_{22}\right)\left[\delta\left(e_{1}\right)+\delta\left(b_{12}\right)\right]\left(a_{12}+c_{22}\right) } \\
& +\left(a_{12}+c_{22}\right)\left(e_{1}+b_{12}\right)\left[\delta\left(a_{12}\right)+\delta\left(c_{22}\right)\right]+\left[\delta\left(e_{1}\right)+\delta\left(a_{12}\right)\right] b_{12}\left(e_{1}+a_{12}\right) \\
& +\left(e_{1}+a_{12}\right) \delta\left(b_{12}\right)\left(e_{1}+a_{12}\right)+\left(e_{1}+a_{12}\right) b_{12}\left[\delta\left(e_{1}\right)+\delta\left(a_{12}\right)\right] \\
=\quad & \delta\left[\left(e_{1}+a_{12} c_{22}\right) e_{1}\left(e_{1}+a_{12}+c_{22}\right)\right]+\delta\left[\left(e_{1}+c_{22}\right) b_{12}\left(e_{1}+c_{22}\right)\right] \\
+ & \delta\left[\left(a_{12}+c_{22}\right)\left(e_{1}+b_{12}\right)\left(a_{12}+c_{22}\right)\right]+\delta\left[\left(e_{1}+a_{12}\right) b_{12}\left(e_{1}+a_{12}\right)\right] \\
=\quad & \delta\left(e_{1}+a_{12}\right)+\delta\left(b_{12} c_{22}\right) \\
= & \delta\left(e_{1}\right)+\delta\left(a_{12}\right)+\delta\left(b_{12} c_{22}\right) .
\end{aligned}
$$

Notice that in the fifth equality we use the facts that

$$
0=\delta\left(a_{12} b_{12} a_{12}\right)=\delta\left(a_{12}\right) b_{12} a_{12}+a_{12} \delta\left(b_{12}\right) a_{12}+a_{12} b_{12} \delta\left(a_{12}\right)
$$


and

$$
\begin{aligned}
0= & \delta\left[\left(a_{12}+c_{22}\right)\left(e_{1}+b_{12}\right)\left(a_{12}+c_{22}\right)\right] \\
= & \delta\left(a_{12}+c_{22}\right)\left(e_{1}+b_{12}\right)\left(a_{12}+c_{22}\right) \\
& +\left(a_{12}+c_{22}\right) \delta\left(e_{1}+b_{12}\right)\left(a_{12}+c_{22}\right) \\
& +\left(a_{12}+c_{22}\right)\left(e_{1}+b_{12}\right) \delta\left(a_{12}+c_{22}\right) \\
= & {\left[\delta\left(a_{12}\right)+\delta\left(c_{22}\right)\right]\left(e_{1}+b_{12}\right)\left(a_{12}+c_{22}\right) } \\
& +\left(a_{12}+c_{22}\right)\left[\delta\left(e_{1}\right)+\delta\left(b_{12}\right)\right]\left(a_{12}+c_{22}\right) \\
& +\left(a_{12}+c_{22}\right)\left(e_{1}+b_{12}\right)\left[\delta\left(a_{12}\right)+\delta\left(c_{22}\right)\right] .
\end{aligned}
$$

(2) The proof is similar to that of (1), so we omit it.

Lemma 3.3. $\delta$ is additive on $R_{12}$.

Proof. We need to show that

$$
\delta\left(a_{12}+b_{12}\right)=\delta\left(a_{12}\right)+\delta\left(b_{12}\right)
$$

holds true for any $a_{12}, b_{12} \in R_{12}$.

For any $x_{1 j} \in R_{1 j}, j=1,2$, from

$$
\delta\left[x_{1 j}\left(a_{12}+b_{12}\right) x_{1 j}\right]=0=\delta\left(x_{1 j} a_{12} x_{1 j}\right)+\delta\left(x_{1 j} b_{12} x_{1 j}\right)
$$

we can get

This implies that

$$
x_{1 j}\left[\delta\left(a_{12}+b_{12}\right)-\delta\left(a_{12}\right)-\delta\left(b_{12}\right)\right] x_{1 j}=0 .
$$

$$
\left[\delta\left(a_{12}+b_{12}\right)-\delta\left(a_{12}\right)-\delta\left(b_{12}\right)\right]_{11}=\left[\delta\left(a_{12}+b_{12}\right)-\delta\left(a_{12}\right)-\delta\left(b_{12}\right)\right]_{21}=0 .
$$

Similarly, by considering

$$
\delta\left[x_{22}\left(a_{12}+b_{12}\right) x_{22}\right]=0=\delta\left(x_{22} a_{12} x_{22}\right)+\delta\left(x_{22} b_{12} x_{22}\right)
$$

for any $x_{22} \in R_{22}$, we can conclude that $\left[\delta\left(a_{12}+b_{12}\right)-\delta\left(a_{12}\right)-\delta\left(b_{12}\right)\right]_{22}=0$.

We now show that $\left[\delta\left(a_{12}+b_{12}\right)-\delta\left(a_{12}\right)-\delta\left(b_{12}\right)\right]_{12}=0$. For any $x_{21} \in R_{21}$, on one hand,

$$
\begin{aligned}
& \delta\left[x_{21}\left(a_{12}+b_{12}\right) x_{21}\right] \\
= & \delta\left(x_{21}\right)\left(a_{12}+b_{12}\right) x_{21}+x_{21} \delta\left(a_{12}+b_{12}\right) x_{21}+x_{21}\left(a_{12}+b_{12}\right) \delta\left(x_{21}\right) .
\end{aligned}
$$

On the other hand, by Lemma $3.2(2)$,

$$
\begin{aligned}
& \delta\left[x_{21}\left(a_{12}+b_{12}\right) x_{21}\right] \\
= & \delta\left(x_{21} a_{12} x_{21}+x_{21} b_{12} x_{21}\right) \\
= & \delta\left(x_{21} a_{12} x_{21}\right)+\delta\left(x_{21} b_{12} x_{21}\right) \\
= & \delta\left(x_{21}\right) a_{12} x_{21}+x_{21} \delta\left(a_{12}\right) x_{21}+x_{21} a_{12} \delta\left(x_{21}\right) \\
& +\delta\left(x_{21}\right) b_{12} x_{21}+x_{21} \delta\left(b_{12}\right) x_{21}+x_{21} b_{12} \delta\left(x_{21}\right) .
\end{aligned}
$$

Then we can get

$$
x_{21}\left[\delta\left(a_{12}+b_{12}\right)-\delta\left(a_{12}\right)-\delta\left(b_{12}\right)\right] x_{21}=0,
$$

which leads to $\left[\delta\left(a_{12}+b_{12}\right)-\delta\left(a_{12}\right)-\delta\left(b_{12}\right)\right]_{12}=0$. This completes the proof.

Similarly, we have

Lemma 3.4. $\delta$ is additive on $R_{21}$. 
Lemma 3.5. $\delta$ is additive on $R_{11}$.

Proof. Let $a_{11}$ and $b_{11}$ be arbitrary elements of $R_{11}$. By considering $\delta\left[x_{i j}\left(a_{11}+\right.\right.$ $\left.\left.b_{11}\right) x_{i j}\right], \delta\left(x_{i j} a_{11} x_{i j}\right)$, and $\delta\left(x_{i j} b_{11} x_{i j}\right)$ for the cases of $i \neq j$ and $i=j=2$ respectively, one can easily get that

$$
\begin{aligned}
& {\left[\delta\left(a_{11}+b_{11}\right)-\delta\left(a_{11}\right)-\delta\left(b_{11}\right)\right]_{12}=0} \\
& {\left[\delta\left(a_{11}+b_{11}\right)-\delta\left(a_{11}\right)-\delta\left(b_{11}\right)\right]_{21}=0} \\
& {\left[\delta\left(a_{11}+b_{11}\right)-\delta\left(a_{11}\right)-\delta\left(b_{11}\right)\right]_{22}=0 .}
\end{aligned}
$$

In order to complete the proof, we need to show that

$$
\left[\delta\left(a_{11}+b_{11}\right)-\delta\left(a_{11}\right)-\delta\left(b_{11}\right)\right]_{11}=0 .
$$

We now claim that, for any $r_{11} \in R_{11}$ and $x_{12} \in R_{12}$,

(3.1) $\delta\left(r_{11} x_{12}\right)=\delta\left(e_{1}\right) r_{11} x_{12}+e_{1} \delta\left(r_{11}\right) x_{12}+r_{11} \delta\left(x_{12}\right)+\delta\left(x_{12}\right) r_{11}+x_{12} \delta\left(r_{11}\right) e_{1}$.

Indeed, by Lemma 3.1,

$$
\begin{aligned}
& \delta\left(r_{11}\right)+\delta\left(r_{11} x_{12}\right) \\
= & \delta\left(r_{11}+r_{11} x_{12}\right) \\
= & \delta\left[\left(e_{1}+x_{12}\right) r_{11}\left(e_{1}+x_{12}\right)\right] \\
= & \delta\left(e_{1}+x_{12}\right) r_{11}\left(e_{1}+x_{12}\right)+\left(e_{1}+x_{12}\right) \delta\left(r_{11}\right)\left(e_{1}+x_{12}\right)+\left(e_{1}+x_{12}\right) r_{11} \delta\left(e_{1}+x_{12}\right) \\
= & \delta\left(r_{11}\right)+\delta\left(e_{1}\right) r_{11} x_{12}+e_{1} \delta\left(r_{11}\right) x_{12}+r_{11} \delta\left(x_{12}\right)+\delta\left(x_{12}\right) r_{11}+x_{12} \delta\left(r_{11}\right) e_{1} .
\end{aligned}
$$

Note that in the last equality we are using the facts that

$$
\delta\left(r_{11}\right)=\delta\left(e_{1} r_{11} e_{1}\right)=\delta\left(e_{1}\right) r_{11} e_{1}+e_{1} \delta\left(r_{11}\right) e_{1}+e_{1} r_{11} \delta\left(e_{1}\right)
$$

and

$$
0=\delta\left(x_{12} r_{11} x_{12}\right)=\delta\left(x_{12}\right) r_{11} x_{12}+x_{12} \delta\left(r_{11}\right) x_{12}+x_{12} r_{11} \delta\left(x_{12}\right) .
$$

Replacing $r_{11}$ in Equation (3.1) with $a_{11}+b_{11}, a_{11}$, and $b_{11}$ respectively, one can get

$$
\begin{aligned}
& \delta\left[\left(a_{11}+b_{11}\right) x_{12}\right] \\
=\quad & \delta\left(e_{1}\right)\left(a_{11}+b_{11}\right) x_{12}+e_{1} \delta\left(a_{11}+b_{11}\right) x_{12}+\left(a_{11}+b_{11}\right) \delta\left(x_{12}\right) \\
+ & \delta\left(x_{12}\right)\left(a_{11}+b_{11}\right)+x_{12} \delta\left(a_{11}+b_{11}\right) e_{1}
\end{aligned}
$$

and, also by Lemma 3.3,

$$
\begin{aligned}
& \delta\left[\left(a_{11}+b_{11}\right) x_{12}\right] \\
= & \delta\left(a_{11} x_{12}\right)+\delta\left(b_{11} x_{12}\right) \\
=\quad & \delta\left(e_{1}\right) a_{11} x_{12}+e_{1} \delta\left(a_{11}\right) x_{12}+a_{11} \delta\left(x_{12}\right)+\delta\left(x_{12}\right) a_{11}+x_{12} \delta\left(a_{11}\right) e_{1} \\
& +\delta\left(e_{1}\right) b_{11} x_{12}+e_{1} \delta\left(b_{11}\right) x_{12}+b_{11} \delta\left(x_{12}\right)+\delta\left(x_{12}\right) b_{11}+x_{12} \delta\left(b_{11}\right) e_{1} .
\end{aligned}
$$

Comparing the above two equalities, we arrive at

$$
\left[\delta\left(a_{11}+b_{11}\right)-\delta\left(a_{11}\right)-\delta\left(b_{11}\right)\right] x_{12}=0 .
$$

It follows from Condition $\left(\mathrm{P} 1^{\prime}\right)$ that

$$
\left[\delta\left(a_{11}+b_{11}\right)-\delta\left(a_{11}\right)-\delta\left(b_{11}\right)\right]_{11}=0 .
$$

Lemma 3.6. $\delta$ is additive on $R_{22}$. 
Proof. With the similar approach as in Lemma 3.5, one can get

$$
\begin{aligned}
& {\left[\delta\left(a_{22}+b_{22}\right)-\delta\left(a_{22}\right)-\delta\left(b_{22}\right)\right]_{11}=0} \\
& {\left[\delta\left(a_{22}+b_{22}\right)-\delta\left(a_{22}\right)-\delta\left(b_{22}\right)\right]_{12}=0,} \\
& {\left[\delta\left(a_{22}+b_{22}\right)-\delta\left(a_{22}\right)-\delta\left(b_{22}\right)\right]_{21}=0 .}
\end{aligned}
$$

To complete the proof, it remains to show that

$$
\left[\delta\left(a_{22}+b_{22}\right)-\delta\left(a_{22}\right)-\delta\left(b_{22}\right)\right]_{22}=0 .
$$

For any $x_{12} \in R_{12}$ and $r_{22} \in R_{22}$, from

$$
x_{12} r_{22}=\left(e_{1}+r_{22}\right) x_{12}\left(e_{1}+r_{22}\right)
$$

one can check that

$$
\delta\left(x_{12} r_{22}\right)=\delta\left(e_{1}\right) x_{12} r_{22}+e_{1} \delta\left(x_{12}\right) r_{22}+r_{22} \delta\left(x_{12}\right) e_{1}+x_{12} \delta\left(r_{22}\right) .
$$

Now, applying Equality (3.2) for $r_{22}=a_{22}+b_{22}, r_{22}=a_{22}$, and $r_{22}=b_{22}$ respectively, we can get

$$
x_{12}\left[\delta\left(a_{22}+b_{22}\right)-\delta\left(a_{22}\right)-\delta\left(b_{22}\right)\right]=0 .
$$

It follows from Condition $\left(\mathrm{P} 2^{\prime}\right)$ that $\left[\delta\left(a_{22}+b_{22}\right)-\delta\left(a_{22}\right)-\delta\left(b_{22}\right)\right]_{22}=0$, which completes the proof.

Applying Lemma 3.1 and Lemmas 3.3 - 3.6, we can get the following result using the same approach as in the proof of Theorem 2.8 .

Theorem 3.7. Suppose $R$ is a ring containing a nontrivial idempotent and satisfies the following conditions:

(P1') If $a_{11} x_{12}=0$ for all $x_{12} \in R_{12}$, then $a_{11}=0$.

$\left(P 2^{\prime}\right)$ If $x_{12} a_{22}=0$ for all $x_{12} \in R_{12}$, then $a_{22}=0$.

(P4) If $x_{i j} a x_{i j}=0$ for all $x_{i j} \in R_{i j}(i, j \in\{1,2\})$, then $a_{j i}=0$.

If a mapping $\delta: R \rightarrow R$ satisfies

$$
\delta(a b a)=\delta(a) b a+a \delta(b)+a b \delta(b) \quad \text { for all } a, b \in R,
$$

then $\delta$ is additive, and hence a Jordan triple derivation.

Note that every Jordan triple derivation on a 2-torsion free semiprime ring is a derivation (see [2]). By Lemma 1.5 we have

Corollary 3.8. Let $R$ be a 2-torsion free semiprime ring containing a nontrivial idempotent and satisfying

(P1') If $a_{11} x_{12}=0$ for all $x_{12} \in R_{12}$, then $a_{11}=0$.

$\left(P \mathscr{2}^{\prime}\right)$ If $x_{12} a_{22}=0$ for all $x_{12} \in R_{12}$, then $a_{22}=0$.

(C3) If $x_{i j} a x_{i j}=0$ for all $x_{i j} \in R_{i j}(i \neq j)$, then $a_{j i}=0$.

If $\delta: R \rightarrow R$ satisfies

$$
\delta(a b a)=\delta(a) b a+a \delta(b)+a b \delta(b) \quad \text { for all } a, b \in R,
$$

then $\delta$ is additive. Moreover, $\delta$ is a derivation.

In particular, applying Lemma 1.6, we get

Corollary 3.9. Let $R$ be a 2-torsion prime ring containing a nontrivial idempotent. If $\delta: R \rightarrow R$ satisfies

$$
\delta(a b a)=\delta(a) b a+a \delta(b)+a b \delta(b) \quad \text { for all } a, b \in R,
$$

then $\delta$ is additive. Moreover, $\delta$ is a derivation. 
Corollary 3.10. Let $\mathcal{A}$ be a standard operator algebra in $X$, where $X$ is a Banach space with $\operatorname{dim} X>1$. If a mapping $\delta: \mathcal{A} \rightarrow \mathcal{A}$ satisfies

$$
\delta(A B A)=\delta(A) B A+A \delta(B) A+A B \delta(A) \quad \text { for all } A, B \in \mathcal{A},
$$

then $\delta$ is additive. Moreover, $\delta$ is an additive derivation.

Acknowledgement: The authors thank the referee who suggests generalizing the original results to a larger class of rings.

\section{REFERENCES}

1. M. Brešar, Jordan derivations on semiprime rings, Proc. Amer. Math. Soc. 104 (1988), 10031006.

2. M. Brešar, Jordan mappings of semiprime rings, J. Algebra 127 (1989), 218-228.

3. P. Li and W. Jing, Jordan elementary maps on rings, Linear Algebra Appl. 382 (2004), 237245.

4. F. Lu, Additivity of Jordan maps on standard operator algebras, Linear Algebra Appl. 357, (2002) 123-131.

5. F. Lu, Jordan triple maps, Linear Algebra Appl. 375 (2003), 311-317.

6. F. Lu, Jordan derivable maps of prime rings, Comm. Algebra (to appear)

7. L. Molnár, Jordan maps on standard operator algebras, in: Functional Equations - Results and Advances, Kluwer Academic Publishers, Dordrecht, 2002.

8. W. S. Martindale III, When are multiplicative mappings additive? Proc. Amer. Math. Soc. 21 (1969), 695-698.

Department of Mathematics \& Computer Science, Fayetteville State University, FAYETTEVILLE, NC 28301

E-mail address: wjing@uncfsu.edu

Department of Mathematics, Suzhou University, Suzhou 215006, P. R. China

E-mail address: fylu@suda.edu.cn 